\title{
The role of interstellar filaments in regulating the star formation efficiency and shaping the initial mass function
}

\author{
Vera Könyves and Philippe André \\ Laboratoire AIM, CEA/DSM-CNRS-Université Paris Diderot, IRFU/Service d'Astrophysique, \\ CEA Saclay, 91191 Gif-sur-Yvette, France \\ emails: vera.konyves@cea.fr, philippe.andre@cea.fr
}

\begin{abstract}
Recent surveys at infrared and submillimeter wavelengths with the Spitzer and Herschel space observatories suggest that star formation in dense molecular gas is governed by essentially the same "laws" in nearby Galactic clouds and distant external galaxies. This raises the possibility of a unified picture for star formation in the Universe from individual-cloud scales to galaxy-wide scales. We summarize the star formation scenario favored by Herschel studies of the nearest molecular clouds of the Galaxy which point to the key role of the quasi-universal filamentary structure pervading the cold interstellar medium.
\end{abstract}

Keywords. stars: formation, ISM: clouds, ISM: structure, ISM: individual objects (Aquila Rift complex), submillimeter

\section{The filamentary interstellar medium}

Interstellar filaments were already seen earlier in molecular clouds (e.g., Schneider \& Elmegreen 1979; Hartmann 2002; Myers 2009), after which Herschel observations not only confirmed their omnipresence, but the ubiquity of filaments also revolutionised our view of star formation in the Galaxy.

The Herschel Gould Belt survey (HGBS, André et al. 2010) observations confirm the omnipresence of filaments in nearby molecular clouds and suggested a close connection between the filamentary structure of the cold ISM and the formation process of prestellar cores (André et al. 2010, Men'shchikov et al. 2010). Besides, other Herschel imaging programs such as HOBYS (Motte et al. 2010), HiGAL (Molinari et al. 2010), and EPoS (Henning et al. 2010) demonstrated the presence and importance of filaments in the interstellar medium. These filaments present a high degree of universality, including their typical inner radial width of $\sim 0.1 \mathrm{pc}$ (e.g., Arzoumanian et al. 2011, Palmeirim et al. 2013, Koch \& Rosolowsky 2015), and they likely play a central role in the star formation process (see for a review André et al. 2014).

\section{Spatial distribution of prestellar cores}

One of the main goals of the HGBS is to elucidate the physical mechanisms responsible for the formation and evolution of prestellar cores in molecular clouds. In the Aquila cloud complex based on Herschel data† we have identified a complete sample of 651 starless cores extracted with the multi-scale, multi-wavelength source finding algorithm getsources (Men'shchikov et al. 2012). Approximately $60 \% \pm 10 \%$ of this sample are

$\dagger$ The Herschel products of the Aquila molecular cloud, including maps and catalogs, can be found at the Herschel Gould Belt Survey Archive, http://gouldbelt-herschel.cea.fr/archives. 

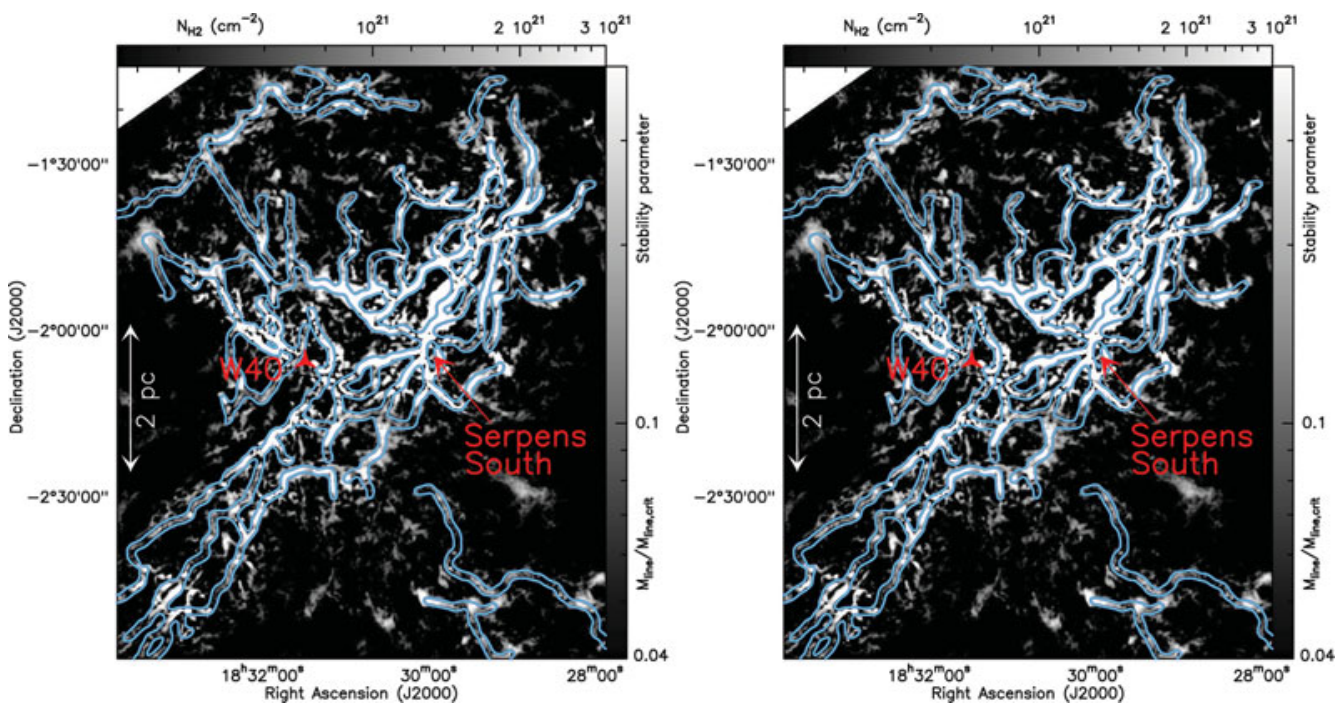

Figure 1. Left: Curvelet component of the Herschel column density map of Aquila, showing the main region in equivalent mass per unit length along the filaments (cf. André et al. 2010), which are likely gravitationally unstable (white region) above half the critical value $M_{\text {line,crit }}=2 c_{\mathrm{s}}^{2} / G$ (Inutsuka \& Miyama 1997). The blue contours outline the $0.1 \mathrm{pc}-$ wide footprints of the filaments (see details in Könyves et al. 2015). Right: Same map as in the left panel with the locations of candidate prestellar cores (blue triangles) and protostellar cores (green up-side-down triangles).

gravitationally bound prestellar cores, which may form (proto)stars in the future. We also detected 58 protostellar cores (Könyves et al. 2010, 2015; Maury et al. 2011).

The comparison of the census of dense prestellar cores and extracted filaments in Aquila suggest their close connection via the core/star formation process. We find that a high fraction of the dense gas mass is in the form of filaments above $A_{\mathrm{V}} \sim 7$. Furthermore, $\sim 90 \%$ of our prestellar cores are located above an equivalent background column density of $\sim 7 \times 10^{21} \mathrm{~cm}^{-2}$, and a similarly high portion of them lie within the densest filaments with supercritical masses per unit length $>16 M_{\odot} / \mathrm{pc}$ (Könyves et al. 2015).

Fig. 1 shows the filamentary structure of the Aquila main field, traced by the curvelet transform component (Starck et al. 2003) of the column density map in the form of an equivalent stability parameter (mass per unit length) along the filaments. In the left panel the filaments are represented by their $0.1 \mathrm{pc}-$-wide footprints traced with DisPerSE (Sousbie 2011), while in the right panel the candidate prestellar cores and protostellar cores are overplotted. The spatial correlation between the densest filaments and the prestellar cores is strong (see details in Könyves et al. 2015).

\section{Prestellar CMF and star formation efficiency}

In Aquila the core mass function of the large population of prestellar cores confirms earlier findings that the shape of the $\mathrm{CMF}$ is very similar to the stellar initial mass function (IMF), and there is a close physical link between the two (Fig. 2/left, Könyves et al. 2015).

The local core formation efficiency at the prestellar core level is high in Aquila, $\epsilon_{\text {core }} \sim 30-40 \%$, and only a small fraction $(\sim 15 \%)$ of the gas mass is in the form 

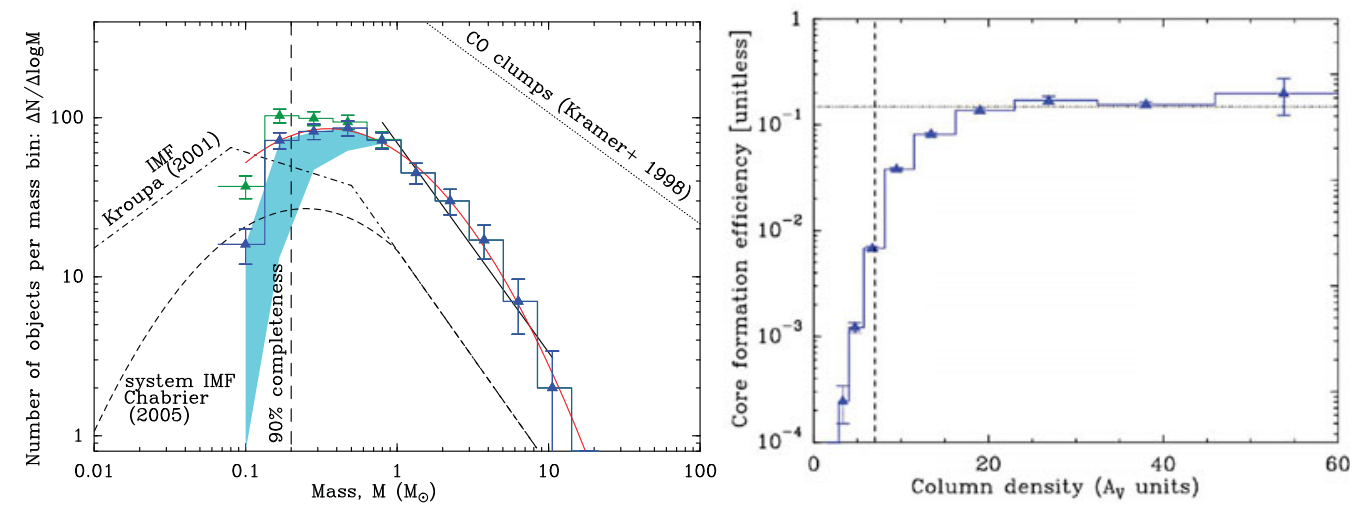

Figure 2. Left: Differential core mass function $(\mathrm{d} N / \mathrm{d} \log M)$ of 651 starless cores (upper green histogram) and 446 candidate prestellar cores (lower blue histogram) identified with Herschel in the Aquila field. The shaded area reflects a fraction of $60 \% \pm 10 \%$ of the starless cores classified as gravitationally bound (see details in Könyves et al. 2015). The lognormal fit to the prestellar cores (red curve) peaks at $\sim 0.5 M_{\odot}$, which is very similar in shape to the IMF of multiple systems (e.g., Chabrier 2005). The power-law fit (black line) has a slope of $-1.33 \pm 0.06$. Right: Histogram of observed differential core formation efficiency (CFE) as a function of background column density in $A_{\mathrm{V}}$ units; $\mathrm{CFE}_{\mathrm{obs}}\left(A_{\mathrm{V}}\right)=\Delta M_{\text {cores }}\left(A_{\mathrm{V}}\right) / \Delta M_{\text {cloud }}\left(A_{\mathrm{V}}\right)$. The vertical dashed line marks the threshold at $A_{\mathrm{V}}^{\mathrm{bg}} \sim 7$. The horizontal dotted line marks the rough asymptotic value of the CFE $\sim 15 \%$, corresponding to a star formation rate per unit gas mass $\sim 5 \times 10^{-8} \mathrm{yr}^{-1}$.

of prestellar cores above $A_{\mathrm{V}} \sim 7$ (André et al. 2014, Könyves et al. 2015). Invoking the typical filament width of $\sim 0.1 \mathrm{pc}$, this observed column density core formation "threshold" corresponds -within a factor of 2- to the threshold above which interstellar filaments become gravitationally unstable (Fig. 1, André et al. 2010, 2014).

In order to support further the threshold for core formation we found from the distribution of background cloud column densities of the prestellar cores (e.g., André et al. 2014, Könyves et al. 2015), we can inspect the observed core formation efficiency (CFE). The right panel of Fig. 2 shows the CFE in Aquila, obtained by dividing the mass in the form of prestellar cores in a given column density bin by the cloud mass observed in the same bin; $\operatorname{CFE}_{\text {obs }}\left(A_{\mathrm{V}}\right)=\Delta M_{\text {cores }}\left(A_{\mathrm{V}}\right) / \Delta M_{\text {cloud }}\left(A_{\mathrm{V}}\right)$. The observations indicate a very steep rise in the range of $A_{\mathrm{V}} \sim 5-15$, below which the prestellar CFE is negligible, and approximately constant, above. Despite of the fact that such realizations of the threshold are not infinitely sharp, we argue for the presence of a physical threshold for prestellar core formation around a fiducial value of $A_{\mathrm{V}}^{b g} \sim 7$ (André et al. 2014, Könyves et al. 2015). With Spitzer, similar threshold has been observed in the spatial distribution of young stellar objects (YSOs) in nearby clouds (Heiderman et al. 2010, Lada et al. 2010, 2012, Evans et al. 2014).

Altogether, our Herschel findings support a picture according to which interstellar filaments and prestellar cores represent two fundamental steps in the formation process of solar-type stars (André et al. 2010, 2014): first, the dissipation of kinetic energy in large-scale MHD flows generates $\sim 0.1 \mathrm{pc}-$-wide filaments in the cold ISM; then the densest filaments -above a critical threshold of $\sim 16 M_{\odot} / \mathrm{pc}$ in mass per unit length- form prestellar cores (and ultimately protostars) by gravitational instability. The peak of the CMF (at $\sim 0.5 M_{\odot}$ in Aquila) may also result primarily from the gravitational fragmentation of marginally critical filaments. 


\section{Filaments-driven universal star formation law in dense gas?}

Our Herschel data in the Aquila Rift cloud complex allows us to directly infer the "efficiency" of the star formation process from the physics of prestellar core formation within filaments (see André et al. 2014, Könyves et al. 2015). The star formation rate per unit mass of dense gas above the threshold is estimated to be SFR/ $M_{\text {dense }} \sim 5_{-2}^{+2} \times$ $10^{-8} \mathrm{yr}^{-1}$ (Fig. 2/right), similar to which was also found independently in nearby clouds by near-/mid infrared studies (Heiderman et al. 2010, Lada et al. 2010, 2012, Evans et al. 2014), and in other galaxies (Gao \& Solomon 2004).

These works use different tracers of the dense gas which can make the comparison somewhat uncertain. We also have to bear in mind that the presented star formation processes may be different in some extreme environments, for instance, in the central molecular zone close to the Galactic center, where star formation is known to be more inefficient above the same density threshold (Longmore et al. 2013).

In spite of the uncertainties, the similar core formation thresholds may be interpreted as the consequence of the ubiquitous filamentary structure of the nearby molecular clouds, manifesting in a quasi-universal "star formation law" within dense gas above the (column) density threshold (see André et al. 2014).

\section{Acknowledgements}

This work has received support from the ERC 'ORISTARS' project (ERC Advanced Grant Agreements no. 291294).

\section{References}

André, P., Men'shchikov, A., Bontemps, S., et al. 2010, A\& A, 518, L102+

André, P., Di Francesco, J., Ward-Thompson, D., et al. 2014, in PPVI, ed. H. Beuther et al., 27

Arzoumanian, D., André, P., Didelon, P., et al. 2011, A\& A, 529, L6

Chabrier, G. 2005, ASSL, 327, 41

Evans, II, N. J., Heiderman, A., \& Vutisalchavakul, N. 2014, ApJ, 782, 114

Gao, Y. \& Solomon, P. M. 2004, ApJ, 606, 271

Hartmann, L. 2002, ApJ, 578, 914

Heiderman, A., Evans, II, N. J., Allen, L. E., et al. 2010, ApJ, 723, 1019

Henning, T., Linz, H., Krause, O., et al. 2010, A\&A, 518, L95

Inutsuka, S.-I. \& Miyama, S. M. 1997, ApJ, 480, 681

Koch, E. W. \& Rosolowsky, E. W. 2015, MNRAS, 452, 3435

Könyves, V., André, P., Men'shchikov, A., et al. 2010, A\&SA, 518, L106+

Könyves, V., André, P., Men'shchikov, A., et al. 2015, A\&SA, in press, arXiv:1507.05926

Kramer, C., Stutzki, J., Rohrig, R., \& Corneliussen, U. 1998, A\&A, 329, 249

Kroupa, P. 2001, MNRAS, 322, 231

Lada, C. J., Lombardi, M., \& Alves, J. F. 2010, ApJ, 724, 687

Lada, C. J., Forbrich, J., Lombardi, M., \& Alves, J. F. 2012, ApJ, 745, 190

Longmore, S. N., Bally, J., Testi, L., et al. 2013, MNRAS, 429, 987

Maury, A. J., André, P., Men'shchikov, et al. 2011, A\& A, 535, A77

Men'shchikov, A., André, P., Didelon, P., et al. 2010, A\&\&A, 518, L103

Men'shchikov, A., André, P., Didelon, P., et al. 2012, A\& A, 542, A81

Molinari, S., Swinyard, B., Bally, J., et al. 2010, A\&AA, 518, L100

Motte, F., Zavagno, A., Bontemps, S., et al. 2010, A\& A, 518, L77

Myers, P. C. 2009, ApJ, 700, 1609

Palmeirim, P., André, P., Kirk, J., et al. 2013, A\&̛A, 550, A38

Schneider, S. \& Elmegreen, B. G. 1979, ApJs, 41, 87

Starck, J. L., Donoho, D. L., \& Candès, E. J. 2003, A\&\&A, 398, 785

Sousbie, T. 2011, MNRAS, 414, 350 